\title{
On the Influence of the Channel Impulsional Response Estimation on the Performance of a Linear Interference Canceller for a DS/CDMA System
}

\author{
P. Díaz, R. Agustí \\ Dept. of Signal Theory and Communications \\ Universitat Politècnica de Catalunya \\ Apdo. 30002,08080 Barcelon, Spain \\ Tel. +3434017201 Fax +3434017200 \\ e-mail pilar@xaloc.upc.es
}

\begin{abstract}
In a Code Division Multiple Access (CDMA) mobile radio communication system, interference from other users is one of the most critical drawbacks due to the lack of perfect orthogonality between user access codes. So far, practical systems have used power control to overcome fading and near/far effects. Another approach which also results in an important improvement of the capacity is the employ of interference cancellers. In this paper a simple linear interference cancellation scheme for coherent BPSK modulation is proposed and analyzed under Rayleigh multipath fading, and in particular, the influence of the channel impulsional response estimation on the performance of the cancellation scheme has been assessed.
\end{abstract}

\section{INTRODUCTION}

In the mobile communication systems of next generation, a substantial increase in capacity will be one of the most important features with regard to current systems. Compensating for the near/far effect is critical for the satisfactory performance of a DS/CDMA radio system. Multiuser detectors and interference cancellers are able to mitigate fading and near/far effects, but also have the more fundamental potential of significantly raising capacity by cancelling multiple access interference.

Various optimal and sub-optimal multiuser demodulation schemes have been investigated. The optimal demodulator for signals that share the same channel in time and frequency as happens in CDMA systems can be found in [1] and is based on a multiuser joint detection scheme. This receiver is, however, too complex to be considered in a practical mobile communication system since its complexity grows exponentially with the number of simultaneous users to be detected. This has motivated the search for practical schemes with performances close to that of the optimal receiver.

The performance of a simple interference cancellation scheme (proposed in [2]) whose complexity only grows linearly with the number of simultaneous users is presented in this paper for a coherent BPSK modulation and under Rayleigh multipath fading channel. To perform interference cancellation, estimation of various parameters of the received signal as channel impulsional response (amplitude and phase) and transmitted bit is essential. In this paper a scheme for such a purpose is proposed and its influence on the canceller performance is analyzed under indoor environment conditions.

In section 2, a synchronous DS/CDMA system using interference cancellation is described for coherent BPSK signalling. In section 3 , a scheme is proposed for parameter estimation in conjunction with a data frame structure. In section 4, the performance of the interference cancellation scheme for the up-link (mobile to base) is discussed under Rayleigh multipath fading in an indoor environment. Finally, some conclusions are drawn in section 5 .

\section{SYSTEM MODEL}

A synchronous DS/CDMA cellular system using Gold codes as spreading sequences has been considered because of the good behaviour of these sequences in terms of their periodic cross-correlation. To implement in practice a synchronous access of all the users at the base station, a simple scheme based on a synchronism structure of low complexity described in [3] is proposed. This scheme is illustrated in Figure 1.

In reception, cancellation is carried out at the base 
station before combining coherently the different propagation paths with a Rake structure. The interference canceller is shown in Figure 2.

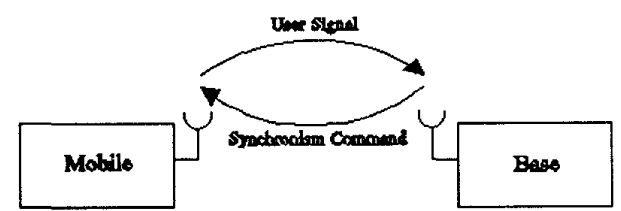

Figure 1. Synchronism scheme for a DS/CDMA synchronous system.

Let $c_{k}(t)$ be the Gold sequence assigned to the $k$-th user, $L$ the number of propagation paths at the receiver input, $M$ the number of arms at the Rake receiver, $K$ the number of simultaneous users in a given cell and $S$ the average received power in absence of multipath.

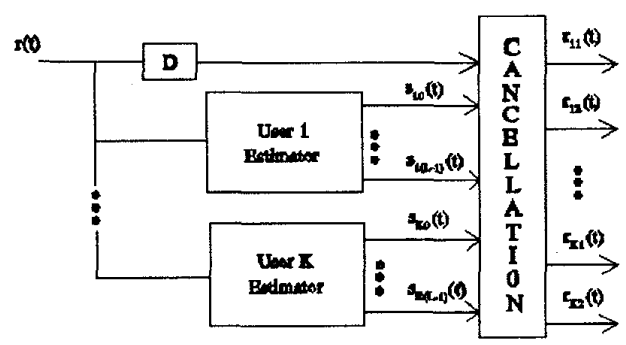

Figure 2. Description of the interference canceller.

Let us see how the cancellation scheme works. The "user $\mathbf{k}$ estimator" module in Figure 2 obtains the signal $s_{\mathrm{ln}}(t)(n=0, \ldots, L-1)$ from the received signal, $r(t)$, as follows:

$$
s_{k n}(t)=\sum_{i=-\infty}^{\infty} \sqrt{2 S} \hat{\alpha}_{k n} c_{k}(t-n T) \hat{d}_{k}^{i} r e c t_{T_{b}}\left(t-i T_{b}\right)
$$

where $\hat{\alpha}_{\mathrm{ln}}$ and $\hat{\mathrm{d}}_{\mathrm{k}}{ }^{\mathrm{i}}$ are respectively the estimate of the $\mathrm{n}$-th propagation path complex amplitude and the estimate of the $\mathrm{i}$-th transmitted bit, both corresponding to the $\mathrm{k}$-th user, and where rect $_{T}(t)$ represents a square waveform of duration $\mathrm{T}$.

Let $n(t)$ represent the interference generated by other users in the adjacent cells. When a perfect synchronism between users within the same cell is assumed at the base station, the signal $\left.r_{j m}(t)(m=1, \ldots, M)\right)$ corresponding to the $\mathrm{j}$-th user at the output of the cancellation module is given by the expression:

$$
\begin{aligned}
& r_{m}(t)=\sqrt{2 S}\left[\sum_{i=-\infty}^{\infty} \alpha_{j n} c_{j}(t-m T) d_{j}^{i} r e c t_{T_{j}}\left(t-i T_{b}\right)+\right. \\
& \sum_{\substack{n=0 \\
n \neq m}}^{L-1} \sum_{\substack{j=-\infty \\
\infty}}^{\infty}\left(\alpha_{j m} d_{j}^{i}-\hat{\alpha}_{j m} \hat{d}_{j}\right) c_{j}(t-n T) \operatorname{rect} t_{T_{b}}\left(t-i T_{b}\right)+ \\
& \left.\sum_{\substack{k=1 \\
k \neq j}}^{R} \sum_{n=0}^{L-1} \sum_{i=-\infty}^{\infty}\left(\alpha_{k n} d_{k}^{i}-\hat{\alpha}_{k n} \hat{d} k_{k}\right) c_{k}\left(t-n T_{j}\right) r e c t_{T_{v}}\left(t-i T_{b}\right)\right]+n(t)
\end{aligned}
$$

These signals are coherently combined by a Rake receiver before decision.

If the canceller was provided with perfect estimates of the channel impulsional response and the transmitted bit

$$
\begin{gathered}
\hat{d}_{k}^{i}=d_{k}^{i} \\
\hat{\alpha}_{k n}=\alpha_{k n}
\end{gathered}
$$

the expression (2) would be as follows:

$$
r_{j m}(t)=\sum_{i=-\infty}^{\infty} \sqrt{2 S} \alpha_{j m} c_{j}(t-m T) d_{j}^{i} \operatorname{rect} t_{T_{j}}\left(t-i T_{b}\right)+n(t)
$$

That is, in a cellular mobile system limited by interference, a linear canceller such as that proposed here would ideally be limited in capacity by the interfering signals from adjacent cells.

In a practical system, however, the channel impulsional response and the transmitted bit must be estimated so that the estimates obtained for being used in the canceller are not perfect. For the cancellation scheme described in Figure 2, we propose to employ a matched filter to the Gold sequence to estimate the channel impulsional response and a Rake structure to estimate the transmitted bit.

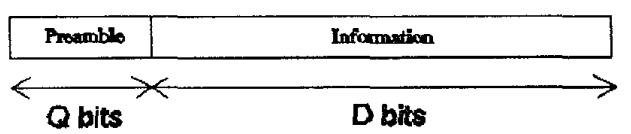

Figure 3. Data frame estructure.

If both estimates are computed from the received signal, $r(t)$, the design of a data frame is needed to allow obtaining independently both estimates. The data structure that has been considered is shown in Figure 3. During the reception of the preamble, the channel impulsional response is obtained from the matched filter designed for 
this purpose, whereas the transmitted bit is estimated during the reception of the information block from the channel estimate obtained in the preamble.

\section{SYSTEM DESIGN}

In the design of parameters $Q$ and $D$, two constraints must be taken into account:

1. The frame efficiency, defined as $D /(Q+D)$, must be maximized. That is, for a given value of $Q$, D should be as large as possible from an efficiency point of view.

2. Due to the temporal variations of the mobile channel, D cannot be as large as we wish.

In order to dimension the frame parameters, a DS/CDMA system with interference cancellation has been simulated. The overall simulated chain is shown in Figure 4, where $s_{T k}(t)$ represents the complex envelope of the signal transmitted by the $k$-th mobile:

$$
s_{T k}(t)=\sum_{i=-\infty}^{\infty} \sqrt{2 S} c_{k}(t) d_{k}^{i} r e c t_{T_{b}}\left(t-i T_{b}\right)
$$

The set of Gold codes assigned to each user, $c_{k}(t)$ $(k=1, \ldots, K)$, has been obtained from two PN sequences of length $N=127$ chips, generated from the characteristic polynomials 211 and 277 (octal) respectively [4].

Let $h_{k}(t)$ be the low-pass equivalent impulsional response of the channel for the $k$-th user:

$$
h_{k}(t)=\sum_{n=0}^{L-1} \alpha_{k n} \delta(t-n T)
$$

The channel has been modelled with an exponential Power Delay Profile (PDP), with a Delay Spread normalised to the bit interval equal to $D_{s n}=0.01$, as usual in an indoor environment.

Concerning the low-pass equivalent Doppler Power Spectrum of each channel propagation path, the classical model [5], given by the following expression

$$
S(f)=\frac{A}{\sqrt{1-\left(\frac{f}{f_{d}}\right)^{2}}} \quad-f_{d}<f<f_{d}
$$

has been employed, where $f_{d}$ is the maximum Doppler frequency. $f_{d} T_{b}=0.0005$ has been set to model the slow channel variations in indoor environments.

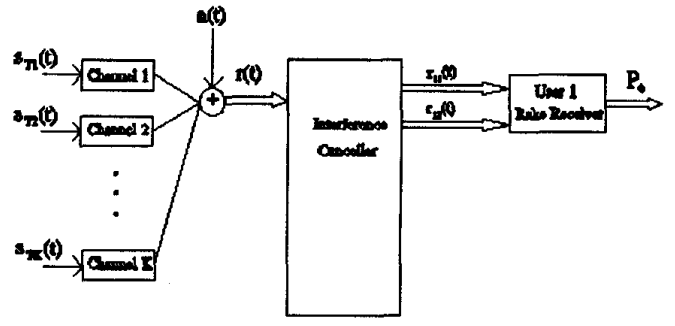

Figure 4. Block diagram of the simulated DS/CDMA

synchronous system.

In order to dimension the parameter $\mathrm{D}$, an ideal system with perfect channel estimates at the beginning of the information block has been considered. For the sake of simplicity, the number of simultaneous users in every cell and the number of arms in the Rake structure have been fixed to $K=5$ and $M=1$ respectively.

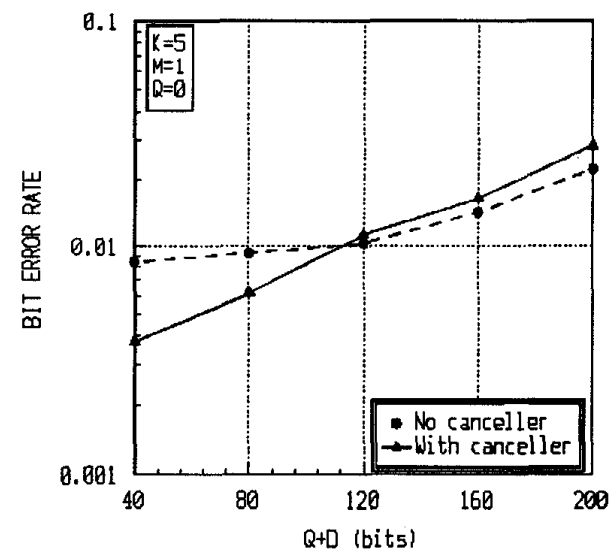

Figure 5. Bit Error Rate for an interference canceller with

perfect channel estimates.

In Figure 5, the results obtained from the simulation for coherent BPSK are represented. They show that system performance degrades as long as $D$ increases when an interference cancellation technique is employed, even for slow-variant mobile channels. This result is sensible given that the estimation error gets greater for those bits located further from the preamble.

A clear conclusion can be drawn from this fact, that is, the need to adapt the channel impulsional response estimate to time variations during the reception of information bits when an interference cancellation scheme is employed. 


\section{SYSTEM PERFORMANCES}

Given the need to adapt the channel estimate for the canceller, the output of the matched filter to the Gold sequence has been used. In order to improve the quality of this estimate by decreasing its jitter, the output of the matched filter has been averaged during $Q$ bits as follows:

$$
\hat{\alpha}_{k n}^{l}=\frac{1}{Q} \sum_{q=i-Q+1}^{i} \hat{\alpha}_{k n}^{q}
$$

where $\alpha_{\mathrm{ln}}{ }^{i}$ is the estimate provided to the canceller at the $i$-th bit interval.

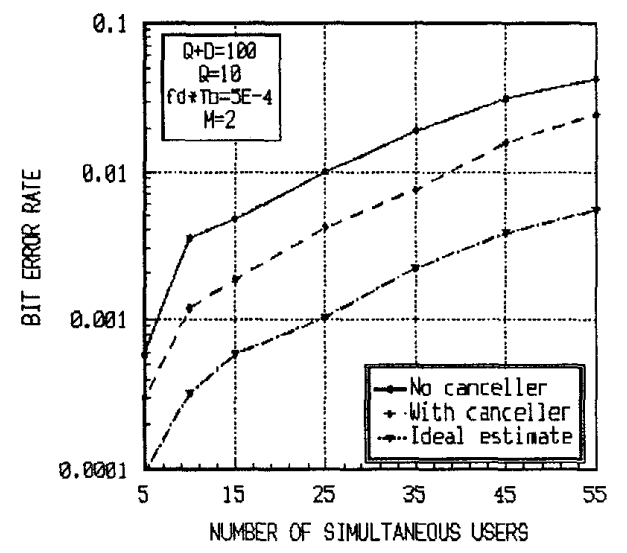

Figure 6. Bit Error Rate at the canceller output for coherent BPSK.

It can be shown from simulations that $Q=10$ and $D=90$ bits are optimal for a channel Doppler frequency normalised equal to $f_{d} T_{b}=0.0005$. Figure 6 shows the system performance as a function of the number of simultaneous users per cell for these values and for a Rake structure made up of two arms $(M=2)$. From the results shown in Figure 6, it can be pointed out that this scheme supports a capacity greater than that supported by a conventional spread-spectrum receiver. When channel coding is used, the capacity improvement is even greater, as shown in Figure 7 for a simple block code (Hamming $(7,4)$ ).

For comparison purposes, it has also been included in Figure 6 a third curve that shows the system performance when the canceller is provided with perfect channel estimates. It points out that further investigation on channel impulsional response estimate may be needed to take total advantage of the canceller capability as long as improvement in capacity is concerned.

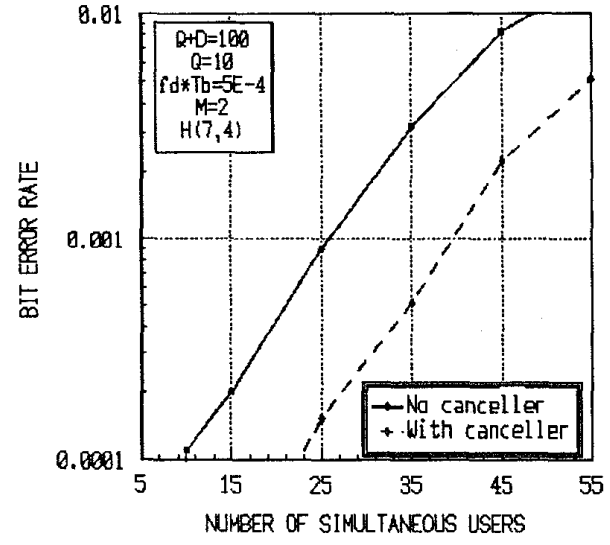

Figure 7. Bit Error Rate at the output of the Hamming $(7,4)$

decoder for coherent BPSK

\section{CONCLUSIONS}

A simple linear canceller for operation at the base station has been analyzed, which provides an important improvement in capacity in indoor environments with regard to the conventional receiver. In particular, the important influence of the channel estimation in the canceller performance has been assessed and a data frame structure in conjunction with a scheme for estimation in slow-variant channels have been proposed for a suitable behaviour of the canceller.

\section{REFERENCES}

[1] S. Verdú, "Minimum Probability of Error for Asynchronous Gaussian Multiple-Access Channels", IEEE Trans. on Information Theory, Vol. IT-32, January 1986.

[2] P. Díaz, R. Agustí, "Analysis of a Linear Interference Canceller in Cellular DS/CDMA Systems", in Proceedings of the 43rd IEEE VTC, June 1994.

[3] P. Díaz, D. Henche, R. Agustí, "A PN Code Delay Estimator Based on the Extended Kalman Filter for a DS/CDMA Cellular System", in Proceedings of the 4th International Symposium on PIMRC, September 1993.

[4] R. de Gaudenzi, C. Elia, R. Viola, "Bandlimited Quasi-Synchronous CDMA: A Novel Satellite Access Technique for Mobile and Personal Communication Systems", IEEE Joumal on Selected Areas in Communications, Vol. 10, No. 2, February 1992.

[5] Cost 207, "Digital Land Mobile Radio Communications", Information Technologies and Sciences (CEC), 1989. 